\title{
COLlaborative WORK BETWEEN HUMAN AND INDUSTRIAL ROBOT IN MANUFACTURING BY ADVANCED SAFETY MONITORING SYSTEM
}

\author{
Vladimir Kuts, Martins Sarkans, Tauno Otto, Toivo Tähemaa
}

Tallinn University of Technology, School Of Engineering, Department of Mechanical and Industrial Engineering, Ehitajate tee 5, 19086 Tallinn, Estonia
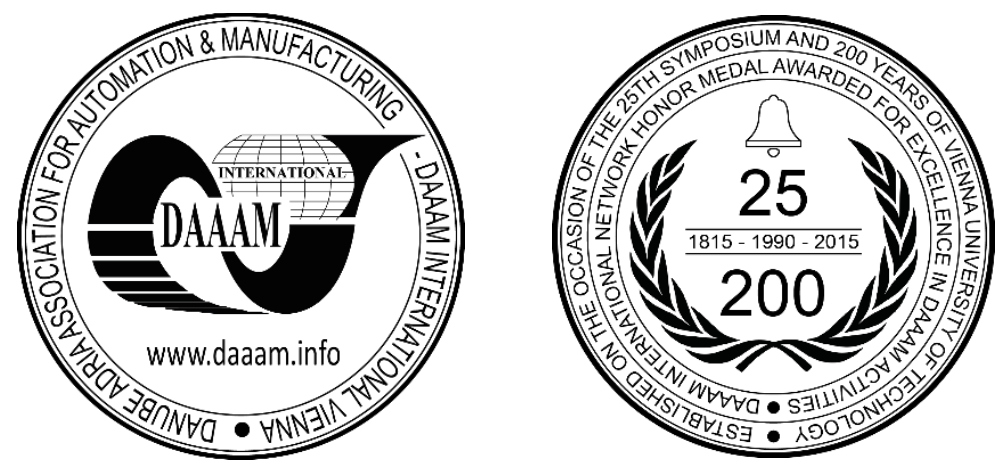

This Publication has to be referred as: Kuts, V[ladimir]; Sarkans, M[artins]; Otto, T[auno] \& Tahemaa, T[oivo] (2017). Collaborative Work Between Human And Industrial Robot In Manufacturing By Advanced Safety Monitoring System, Proceedings of the 28th DAAAM International Symposium, pp.0996-1001, B. Katalinic (Ed.), Published by DAAAM International, ISBN 978-3-902734-11-2, ISSN 1726-9679, Vienna, Austria

DOI: $10.2507 / 28$ th.daaam.proceedings.138

\begin{abstract}
Collaborative work between human and industrial robot in different areas is developing rapidly. High payload industrial robots that can harm humans execute their tasks in separated protected areas and are regulated by safety standards. The main aim of this study was to design experiments, analyze, and model the collaborative work and safety monitoring systems by using small industrial robots with inbuilt power and force limiting. Different methods of collaboration are standardized by the ISO/TS 15066:2016. Human co-work with robots gives us a variety of options that can be used in manufacturing and other related areas. The advanced multi-level monitoring system of co-work processes can reach the highest level of the human safety with minimum robot downtime. Several methods were analyzed to find the optimal solution for the online monitoring processes. As a result, a safety multi-level monitoring system was designed, tested in simulation software with real-room conditions with an industrial robot. Safety multi-level system, as a part of the control and monitoring online system consist of different types of sensors and a microcontroller that can be attached to the Cyber Physical Production System (CPPS). Those control and monitoring methods and processes were implemented in experimental setup in Robot Operating System (ROS).
\end{abstract}

Keywords: collaborative industrial robots; Cyber Physical Production System; Robot Operating System

\section{Introduction}

The collaboration between industrial robots and humans is a widely-discussed topic nowadays. Many research papers have been published in this field, but there are still a few uncovered areas, mostly relating to the devices ranging between collaborative robots and small simple industrial robots. There is also lack of optimized safety systems, which could fulfill all safety requirements and manufacturing needs. 
Different system components, software, and other tools were used in making proper methodologies for studying industrial robot and human collaboration [1], whereas ISO/TS 15066:2016 is the latest recognized standard for this kind of systems. As the development process in the field of collaborative robots is so rapid, we predict that this standard gets updates in the nearest years.

\subsection{Problem statement}

There are two main problems in small industrial robots and human worker collaboration systems:

- Human safety monitoring system control

- Downtime of the industrial robot, while safety system is activated

In the modern robot systems, every large manufacturer has developed its own controller and software where off-line programming (OLP) is done. In this kind of systems, industrial robots are executing their tasks and moving according to the pre-programmed path which is already done before the real work is performed. To gain better control of the movement of the industrial robot it must be possible to affect robot behavior online, while the task is being executed. In this paper, we demonstrate a method for online monitoring and control of the robot with the help of the robot operating system (ROS). In the experimental part of this article, the comparison of the off-line programming (OLP) vs online control, and monitoring method is shown, which was enabled with the new computer technologies and machine vision applications.

\subsection{Background and current situation}

Non-collaborative industrial robots must usually comply with safety requirements described in standards ISO 12100 , ISO 13850, ISO 13855 etc. As there is no need for human intervention during the manufacturing process (i.e. painting, welding, deburring, etc.) strict rules exist for the safety of the human and for emergency halts of the industrial robot. Standards and guidelines describe the allowed speeds, distances, required safety equipment and safety procedures to establish the required safety. When human enters into the workspace of the robot, the process (and the robot) is stopped immediately. To restart the production process, the fault situation must be cleared (worker must leave from the workspace or remove the obstacle) by resetting emergency button outside the cell. As the production processes are growing more complex and interconnected, the human and robot collaboration principles development has become an important issue during the last years. Industrial robots have advantages in areas (processes) where speed, power, repetitiveness, and durability is needed. The human worker can be added into the process to increase agility and flexibility (if the process or product changes rapidly).

Different authors have researched human-robot collaboration topics such as:

- ROS based coordination - For coordination of assembly tasks between human and robot an ROS based software architecture is used. Human and robot tasks are simulated using OLP tool and tasks are recalled using graphical user interface (GUI) thus enabling to separate the tasks for a robot and human operators. [2]

- Human safety - Concept of kinetostatic safety for human-robot collaboration is introduced and its computational methodology is presented. [3]

- Assembly cell - Methodology for task assignment and scheduling for human-robot cooperation (HRC) assembly cell. By using ROS software platform the overall framework for HRC is developed. [4, 5]

- Assembly Factories of the Future (FoF) - Project ROBO-PARTNER is introduced. Its main goal is to develop the integration platform for safe human-robot collaboration (HRC). Different areas like safety, collaboration tasks planning, robot programming, and integration are considered. [6]

- Speed and separation monitoring - Developing a solution for human-robot collaboration in the automotive industry (PSA) for the assembly process. [7]

- Sensors - implementation and integration of different types of sensors to establish required safety for humanrobot collaborations [8]

ISO 15066 standard gives advice for human-robot co-operation safety issues. These are recommended for the development of robot cell solutions. By standard, the workspace is divided into two parts: robot workspace (operating space); and collaborative workspace. Collaborative workspace is defined as "space within the operating space where the robot system (including the workpiece) and a human can perform tasks concurrently during production operation" [9]. As long as the industrial robot operates in its allowed operating space then the general rules of safety are applied (robot stops immediately when someone enters the robot workspace). As robot enters into collaboration workspace, the standard ISO 15066 must be applied. Our research paper concentrates mainly on collaboration workspace issues.

Usually, the robot is programmed by using the teach pendant or offline programming (OLP) method. After the program is simulated in the computer, it is uploaded to the robot controller storage memory. To execute this program on the robot the program is uploaded into program (RAM) memory. During this step, the program syntax is controlled to prevent any faulty code or program. 
This kind of programming approach is quite rigid and usually does not include any human safety factors. The program can be stopped by using the external stop command, additional sensors, safety stop, or a multi-task option. However, in real-life conditions, these are not flexible and consume additional process time. Also, the response of the robot controller can be too slow to halt (or decelerate) the robot before the human gets harmed.

The proposed solution described in this article is to unload the program (connected to the production process) from the robot controller and to convert robot into the client mode for listening only "external" commands from the server. After the conversion, the program can be divided into smaller subprograms and the safety commands and parameters can be added.

Some of the authors have contributed to the robot communication, machine vision, and robot trajectory planning:

- Planning of the trajectory for the robot by using an external camera for this purpose. [10]

- Communication principles between networked robots and remote control solutions are described [11]

- Use of Robot Operating System (ROS) for implementation and programming of mobile robots for process [12]

- Use of computer vision for mobile robots trajectory planning and mapping [13]

Abovementioned concepts can be used as a base for further development also in this case. Although this case includes only one robot in future several robots will be included in this study.

\section{Methodology}

Both of the solutions - robot path planning, and preliminary program - are done in OLP software. The difference can be seen on control and execution level.

\subsection{OLP and online programming comparison}

Many different tools exist for OLP programming - originally all industrial robot manufacturers - ABB, Kuka, Fanuc, Universal Robot, and much more, have their own tools for giving the robot the ability to execute the exact task - welding, measurement, or grabbing depending from the production line/user needs. This way of making a program is nowadays mostly used by users and robotic cell system planners [14]. Generally, the system consists of the robot controller, industrial robot manipulator and a number of sensors. Additional specific components can be added to the system. (See. 1.).

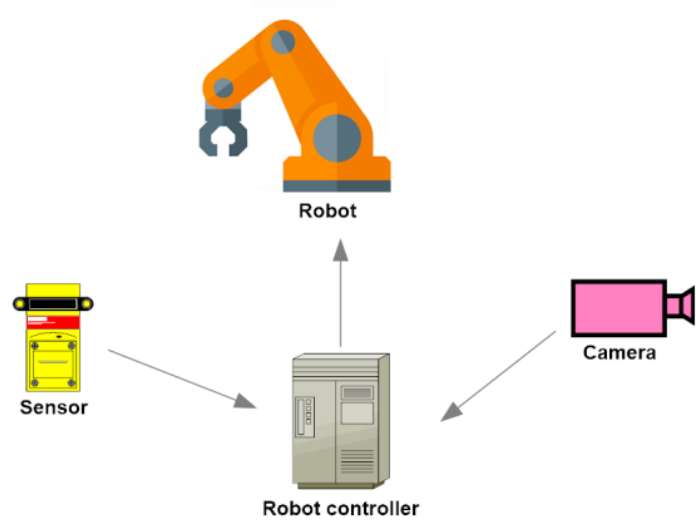

Fig. 1. Robot path executed from OLP

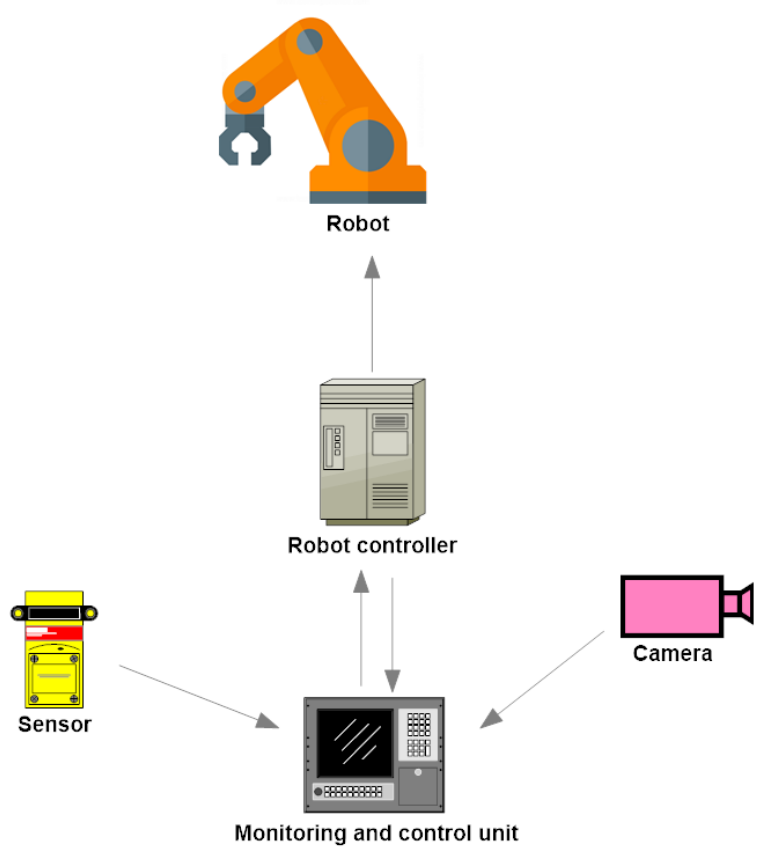

Fig. 2. System with online control

With the online control and monitoring method program for the industrial robot, the path is still programmed by manufacturer's software, but we add an additional control tool, as ROS for example which can be run on a separate controller, or server machine (See Fig. 2.). 
This method gives the system unlimited abilities for:

- $\quad$ process monitoring and control;

- program online modifications;

- automated decision making;

- $\quad$ advanced safety system (security levels); and

- $\quad$ automatic path planning with the camera.

For example, if we have rapid code command which consists of:

- MoveL, which is linear movement command

- $\mathrm{XYZ}$ - coordinates in the system

- $\mathrm{V}$ - speed in $\mathrm{mm} / \mathrm{sec}$

- $\mathrm{Z}$ - curve on path while moving through coordinates

This is enough to execute the task from point A to point B (see Fig. 3.). However, to gain additional artificial intellect to our robot program we need to add an additional factor to the code. Fully effective usage of this can be done via online monitoring and control tool.

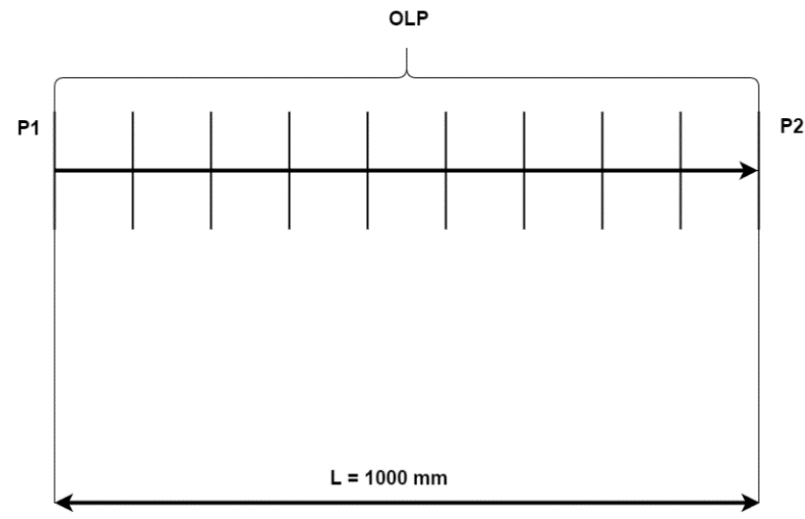

\section{Research}

Fig. 3. Pre-programmed robot path

\subsection{Design of Experiment}

An additional factor in our system is security level of each trajectory part. As in the previous study with OLP, we have 10 coordinates, which must be passed through by the industrial robot according to the pre-done program. By adding the additional factor, it is possible to give to every one of those points a security level on a scale from 1 to 10 , where 10 means "highly secure". So, each step will be monitored with the refresh rate of 0.01 seconds online during the whole program execution progress. If sensor or camera will detect any object in the range of the industrial robot, additional control unit, will decide and give a command to shut down, go to sleep mode, change the path or jump over to the next task. So, as the safety status drops (somebody or something enters the area), the parameter gets a smaller value (0...9) depending on the distance between the object and the duration of the breach. And when the object/human leaves the zone, the program automatically gives permission to proceed with the same step or to go back to the not performed task. On the next round of the program, control unit already remembers where the fault appeared and moves with an exact level of additional notice into that coordinate, and reduces it with every cycle (See Fig.4).

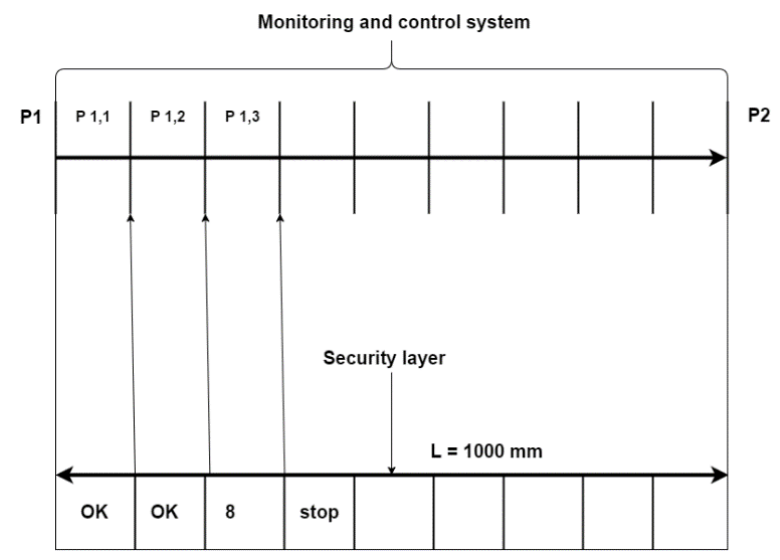

Fig. 4. Robot path with online monitoring 


\subsection{Case study - safety system example}

The example was done using ROS [15] and ABB robot IRB120. Industrial robot and sonar scanner with data about objects, joints location coordinates and physical parameters (See Fig.5) were added to the system.

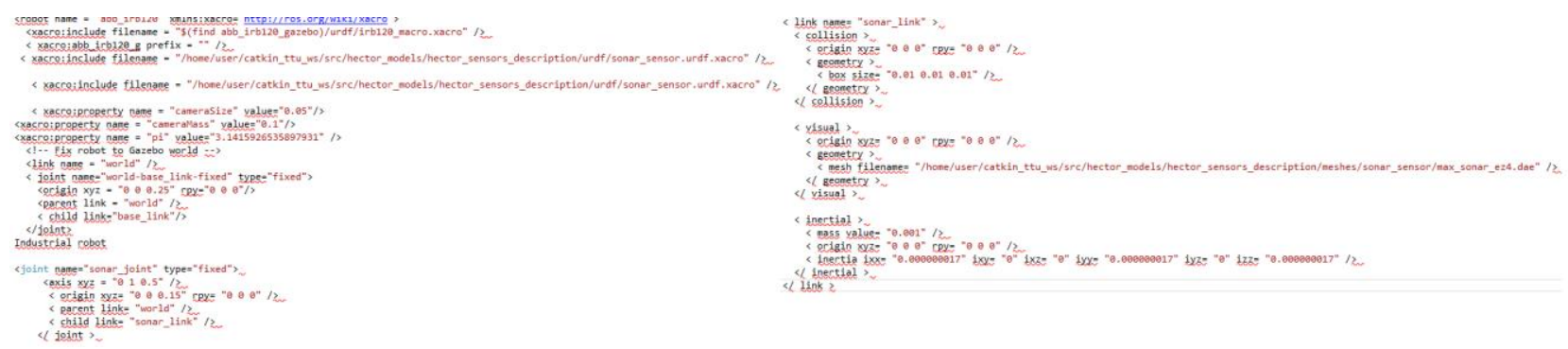

Fig. 5. Sonar scanner parameters

The robot is executing its task. After an object was put into the range of the sonar sensor in the simulation software - the robot stopped executing the task and automatically proceeded with work, when the object was removed (See Fig. 6)

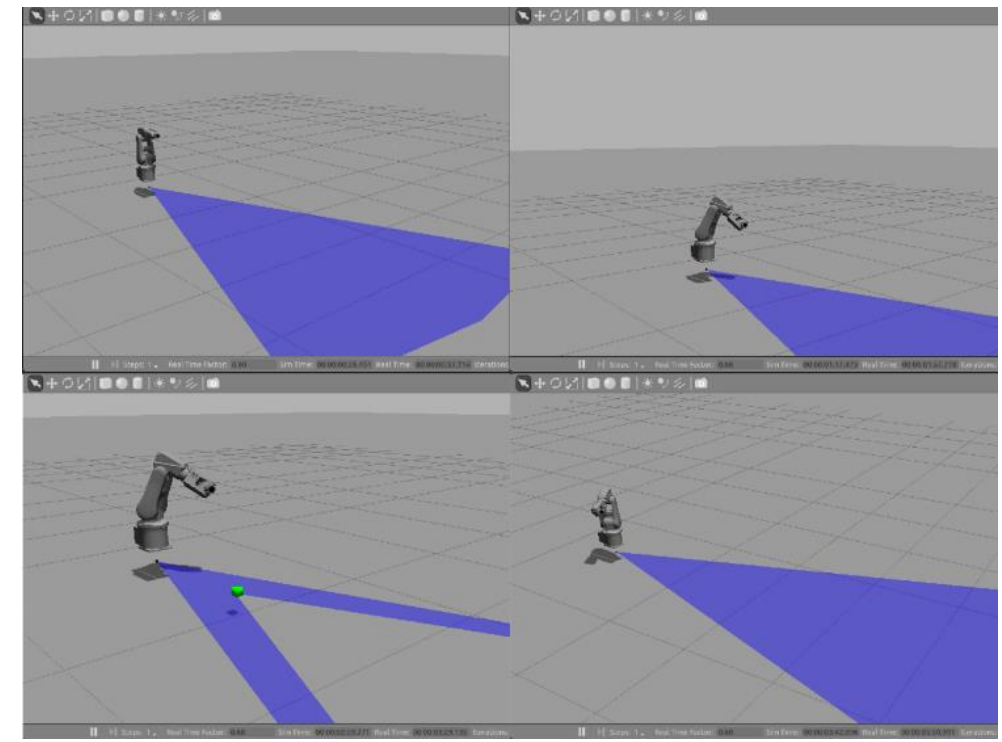

Fig. 6. Case study with safety system

\subsection{Comparison of different methods}

This experiment, comparing OLP and online monitoring methods, has shown that the side control of the robotic path leads to more effective results. While executing the path, additional factors can be added and in most cases, a pre-done program is not needed - according to the commands from the sensors, the robot path execution can be generated online.

This method makes industrial robots more intelligent and gives them the ability to decide about one or another task by themselves. Still, many factors must be considered like movement kinematics and maximum speeds of the robot joints. Considering this and implementing an online monitoring method into manufacturing process will give an unlimited amount of additional features to the manufacturing line. Moreover, first steps to create a tool for online programming in Virtual Reality (VR) were done with the development of real and virtual manufacturing line communication architecture [16], where methods described in this research can be tested.

\section{Discussion}

OLP and online monitoring and control methods were compared and discussed in order to find an optimal way for robot re-programming. Proposed solution based on ROS enables pre-check unattended human intervention and stop the robot cell work until danger of harming the human is de-activated. The methodology was designed for ABB small sized IRB series industrial robot, but the due openness of ROS it can be used also in other manufacturers' industrial robots. With online monitoring of the industrial robot process, there is no limit for the features of the robotic system. The method described in this paper and the case study is only the beginning of the future development of this topic. 
Next steps are:

- Control of different manufacturers' robots with one tool

- Simulation improvements ( reducing response time and increasing flexibility)

- Human presence simulation in the VR

- Flexible robot cell design using VR and simulations tools

In the future, this area should be further investigated and new tools for more precise simulations developed as the human precision simulation using VR tools.

\section{Acknowledgements}

The research was supported by I4MS project SmartIC Robotics - Regional Digital Innovation Hub in Robotics in Estonia. Authors are grateful to the Integrated Engineering students of Tallinn University of Technology - Mohammad Tavassolian, Aleksei Tanjuhhin, and Tengiz Pataraia helping in experiments and simulations.

\section{References}

[1] Vysocky A., Novak P. (2016) Human-Robot Collaboration in Industry. MM (Modern Machinery) Science Journal, June 2016

[2] Tsarouchia P., Makrisa S., MichalosaG., Matthaiakisa A.-S., Chatzigeorgioua X., Athanasatosa A., Stefosa M., Aivaliotisa P., Chryssolourisa G. (2015) ROS based coordination of human-robot cooperative assembly tasks - An industrial case study. Procedia CIRP 37, 2015, $254-259$

[3] Polverini M.P., Zanchettin A.M., Rocco P. (2017) A computationally efficient safety assessment for collaborative robotics applications, Robotics and Computer-Integrated Manufacturing, 46, 2017, 25-37.

[4] Ore F., Vemula B.R., Hanson L., Wiktorsson M. (2016) Human - Industrial Robot Collaboration: Application of Simulation Software for Workstation Optimisation, Procedia CIRP, 44, 2016, 181-186.

[5] Tsarouchi P, Matthaiakis AS, Makris S, Chryssolouris G. (2016): On a human-robot collaboration in an assembly cell, International Journal of Computer Integrated Manufacturing

[6] Michalos G, Makris S, Spiliotopoulos J, Misios I, Tsarouchi P, Chryssolouris G. (2014) ROBO-PARTNER: Seamless Human-Robot Cooperation for Intelligent, Flexible and Safe Operations in the Assembly Factories of the Future, Procedia CIRP 23 ( 2014 ) 71-76

[7] Cherubini A, Passama R, Crosnier A, Lasnier A, Fraisse P. (2016) Collaborative manufacturing with physical human-robot interaction, Robotics and Computer-Integrated Manufacturing 40 (2016) 1-13

[8] Bdiwi M. (2014) Integrated sensors system for human safety during cooperating with industrial robots for handingover and assembling task, Procedia CIRP 23 (2014) $65-70$

[9] ISO/TS 15066:2016 Robots and robotic devices - Collaborative robots (2016)

[10] Katsurin, A. (2016). Planning Trajectory of the Mobile Robot with a Camera, Proceedings of the 26th DAAAM International Symposium, pp.0407-0416, B. Katalinic (Ed.), Published by DAAAM International, ISBN 978-3902734-07-5, ISSN 1726-9679, Vienna, Austria, DOI:10.2507/26th.daaam.proceedings.055

[11] Andreev, V.; Pletenev, P.; Poduraev, Y. (2016). The Method of Network Control of Robotic Systems of Different Models and Manufacturers, Proceedings of the 26th DAAAM International Symposium, pp.0727-0730, B. Katalinic (Ed.), Published by DAAAM International, ISBN 978-3-902734- 07-5, ISSN 1726-9679, Vienna, Austria, DOI:10.2507/26th.daaam.proceedings.101

[12] Khassanov, A.; Krupenkin, A.; Borgul, A. (2014). Control of the Mobile Robots with ROS in Robotics Courses, Proceedings of the 25th DAAAM International Symposium, pp.1475-1484, B. Katalinic (Ed.), Published by DAAAM International, Published by Elsevier in Procedia Engineering 100 (2015) 1475 - 1484.

[13] Gryaznov, N.; Lopota, A. (2014). Computer Vision for Mobile On-Ground Robotics, Proceedings of the 25th DAAAM International Symposium, pp.1376-1380, B. Katalinic (Ed.), Published by DAAAM International, Published by Elsevier in Procedia Engineering 100 (2015) 1376 - 1380.

[14] Kuts, V.; Tähemaa, T.; Otto, T.; Sarkans, M.; Lend, H. (2016). Robot manipulator usage for measurement in production areas. Journal of the Machine Engineering, 16 (1), 57-67.

[15] ROS documentation, Available from: http://wiki.ros.org/ Accessed: 2016-11-25 25.11.2016)

[16] Kuts, V.; Modoni, G. E.; Terkaj, W.; Tähemaa, T.; Sacco, M.; Otto, T. (2017). Exploiting factory telemetry to support Virtual Reality simulation in robotics cell. Augmented Reality, Virtual Reality, and Computer Graphics, 1: 4th International Conference, AVR 2017, Ugento, Italy, June 12-15, 2017. Ed. L. Tommaso De Paolis, P. Bourdot, A. Mongelli. Springer, 212-221. (Lecture Notes in Computer Science; 10324).10.1007/978-3-319-60922-5_16. 